\title{
Using Sydromic Surveillance to Track E-cigarette Related Emergency Department Visits
}

\author{
Jill K. Baber* and Tracy Miller \\ Division of Disease Control, North Dakota Department of Health, Bismarck, ND, USA
}

\section{Objective}

To explore the use of emergency department syndromic surveillance data to identify adverse health events related to electronic cigarettes in order in enhance existing surveillance.

\section{Introduction}

The North Dakota Department of Health (NDDoH) investigated the feasibility of using syndromic surveillance (SyS) data to identify health care visits due to electronic cigarette (e-cigarette) use. E-cigarettes have been associated with injuries and fatalities in all age groups, including young children attracted to the colorful liquid nicotine carriage packaging [1]. Previously, poison control data was the only resource available to the $\mathrm{NDDoH}$ for e-cigarette adverse outcomes surveillance.

\section{Methods}

Data for all visits from June 28, 2014 to June 28, 2015 were downloaded using the BioSense 2.0 SyS analytic tool. Excel was used to identify visits containing key words related to e-cigarettes in linelevel data. We initially searched for visits using variations of the term "e-cigarette." After meeting with NDDoH subject matter experts, we expanded our search to include other related terms: nicotine, clouding, vaping and variations of "electronic nicotine delivery system (ENDS)". No diagnosis codes were used as none refer specifically to e-cigarettes. Visits were identified solely through searching free text chief complaint and triage notes fields. Not all facilities participating in the NDDoH SyS program during this time period submitted free text data.

\section{Results}

Out of 650,069 unique visits, four e-cigarette-related visits were identified in rich-text data fields searching for "E-cig" and "E cig." An additional visit was identified using the search term "nicotine," although this search primarily identified visits including references to nicotine patches. Of the five visits identified, two were poisonings resulting from small children sucking on liquid nicotine cartridges, one referred to eye irritation as a result of accidentally using liquid nicotine as eye drops, and two referred to cardiac issues (chest pain, heart palpitations) after e-cigarette use. Searches including terms "clouding" and "vaping," street terms related to e-cigarettes, did not result in the identification of any additional visits related to e-cigarettes; nor did searches related to ENDS. Poison control data from the same time period yielded two calls related to e-cigarette adverse events.

\section{Conclusions}

It is possible to identify emergency department visits associated with e-cigarette use utilizing SyS data. More visits were identified using SyS data than poison control data, although neither source identified many occurrences of adverse outcomes related to e-cigarettes. E-cig, e cig and nicotine were the most useful search terms, although a search for "nicotine" must exclude the word "patch" to avoid false identifications. The NDDoH receives free-text data for a majority of the visits in our system, but not all facilities submit free-text fields, and the number that did varied over the study period.
Because no drop-down chief complaints or diagnosis codes related to e-cigarettes exist, data from facilities that did not provide free text data were not helpful in identifying e-cigarette-related visits. This investigation emphasizes the need for free text fields when using SyS to investigate emerging issues.

\section{Keywords}

Syndromic Surveillance; Electronic Cigarettes; Injury

\section{References}

1. Hua M, Talbot P. Potential Health Effects of Electronic Cigarettes: A Systematic Review of Case Reports. Prev Med Rep; 2016 Dec;4: 169-178.

\section{*Jill K. Baber}

E-mail: jbaber@nd.gov 